\title{
Antiprotonic helium atomcules
}

\author{
Sébastien Sauge ${ }^{1, a}$ \\ Department of Microelectronics and Information Technology, Royal Institute of Technology (KTH), \\ Electrum 229, SE-16440 Kista, Sweden
}

\begin{abstract}
About $3 \%$ of antiprotons ( $(\overline{\mathrm{p}})$ stopped in helium are long-lived with microsecond lifetimes, against picoseconds in all other materials. This unusual longevity has been ascribed to the trapping of $\overline{\mathrm{p}}$ on metastable bound states in $\overline{\mathrm{p}} \mathrm{He}^{+}$helium atom-molecules thus named atomcules. Apart from their unique dual structure investigated by laser spectroscopy - a near-circular quasi-classical Rydberg atom with $l \sim n-1 \sim 37$ or a special diatomic molecule with a negatively charged $\overline{\mathrm{p}}$ nucleus in high rotational state with $J=l$ - the chemical physics aspects of their interaction with other atoms or molecules constitute an interesting topic for molecular physics. While atomcules may resist to million collisions in helium, molecular contaminants such as $\mathrm{H}_{2}$ are likely to destroy them in a single one, down to very low temperatures. In the Born-Oppenheimer framework, we interpret the molecular interaction obtained by ab initio quantum chemical calculations in terms of classical reactive channels, with activation barriers accounting for the experiments carried out in $\mathrm{He}$ and $\mathrm{H}_{2}$. From classical trajectory Monte Carlo simulations, we show that the thermalization stage strongly quenches initial populations, thus reduced to a recovered $3 \%$ trapping fraction. This work illustrates the pertinence of chemical physics concepts to the study of exotic processes involving antimatter. New insights into the physico-chemistry of cold interstellar radicals are anticipated.
\end{abstract}

\section{Introduction}

About $3 \%$ of antiprotons $(\overline{\mathrm{p}})$ stopped in pure helium survive several microseconds (against a few picoseconds in all other materials). This unusual metastability observed by CERN physicists in 1993 has been ascribed to the the capture of a $\bar{p}$ by an helium atom, followed by ionization of one electron. This exotic system composed of three particles $\left(\alpha, \overline{\mathrm{p}}, \mathrm{e}^{-}\right)$was called atomcule [1] because it can be regarded either as a near-circular quasi-classical Rydberg atom with large angular momentum $l \sim 37$ or, alternatively, as a special diatomic molecule with a negatively charged $\overline{\mathrm{p}}$ nucleus in a low vibrational, high rotational $J=l$ state, see Fig. 1 . The energy level structure of the metastable atomcule, which de-excites through slow radiative transitions, can be accessed by spectroscopy [2]. Measurements are based on resonant de-excitation from a metastable state to an state unstable by Auger emission of the remaining electron, which prompts the $\bar{p}$ to the nucleaus and leads to an annihilation spike on the spectrum. Moreoever, transition wavelengths were remarkably predicted theoretically with a few ppm accuracy [3]. With calculations now incorporating quantum electrodynamics corrections, atomcules thus constitute a benchmark system for validating few-body theories.

a e-mail: ssauge@yahoo.fr 


$$
v_{J}(R)=-\frac{Z}{R}+\frac{J(J+I)}{2 M R^{2}}+e_{J}(R)
$$

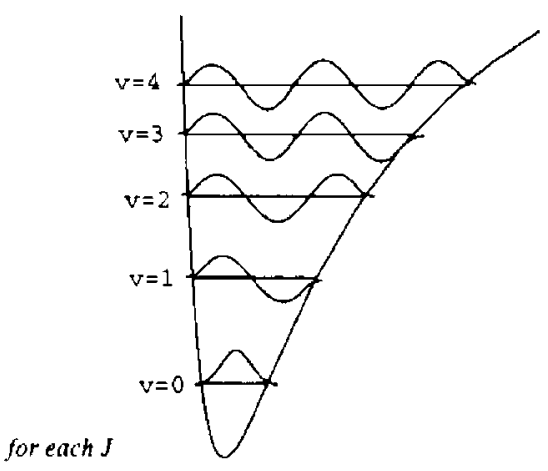

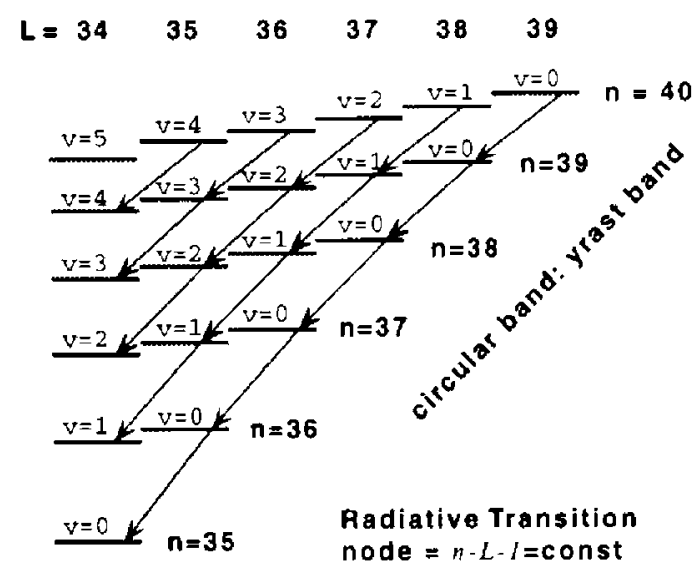

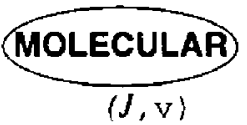

unharmonic rotation vibration

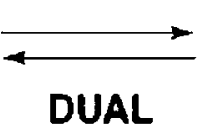

$J=L$

$\mathrm{v}=n-L-I$

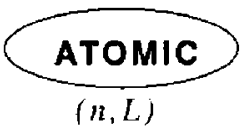

Fig. 1. Dual structure of the antiprotonic atomcule. It can be viewed as a quasi-classical Rydberg atom of typical quantum numbers $(n, L \sim n-1)=(38,37)$, which de-excites through slow radiative transitions conserving the vibrational quantum number $v=n-L-1$. The atomcule can also be considered as a special diatomic molecule with quantum numbers $(v, J)=(n-L-1, L)$, a nucleus of charge -1 , and a strong rotational excitation $(J=L \sim 40)$. Vibrational levels $v=0,1,2 \ldots$ are quantified in the effective potential $V_{J}(R)$.

While the metastability of isolated atomcules is well understood, their collisional quenching remains puzzling. While atomcules resist to millions of collisions in pure helium, molecular contaminants such as $\mathrm{H}_{2}$ destroy them in a single collision, down to very low temperatures. State-resolved decay rates with $\mathrm{H}_{2}$ were measured at $30 \mathrm{~K}$ with geometric collisional cross sections [2], thus imposing severe constraints on theoretical models.

\section{Results}

One of the interesting features of this work was to illustrate the transferability of the usual concepts of chemical physics to the study of exotic processes. In the framework of the Born-Oppenheimer approximation, we calculated the electronic potential of the atomcule interacting with either He or $\mathrm{H}_{2}$ for various configurations of the nuclei, by using standard ab initio quantum chemistry codes. We then averaged the electronic potential over the ro-vibronic wave functions of the nuclei in the most favorable reactive approach. For each state of angular momentum $l$, the reactive channel was then described by the averaged energy as a function of intermolecular separation. As illustrated in Fig. 2 (left part), we attribute the collisional stability of thermalized atomcules in pure helium to the existence of high activation barriers due to Pauli repulsion [4]. Those barriers get lowered with increasing angular momentum values of $l$, accounting for the absence of atomcules with $l>40$ (their initial recoil energy, after capture of the $\overline{\mathrm{p}}$, is sufficient to cross barriers). 

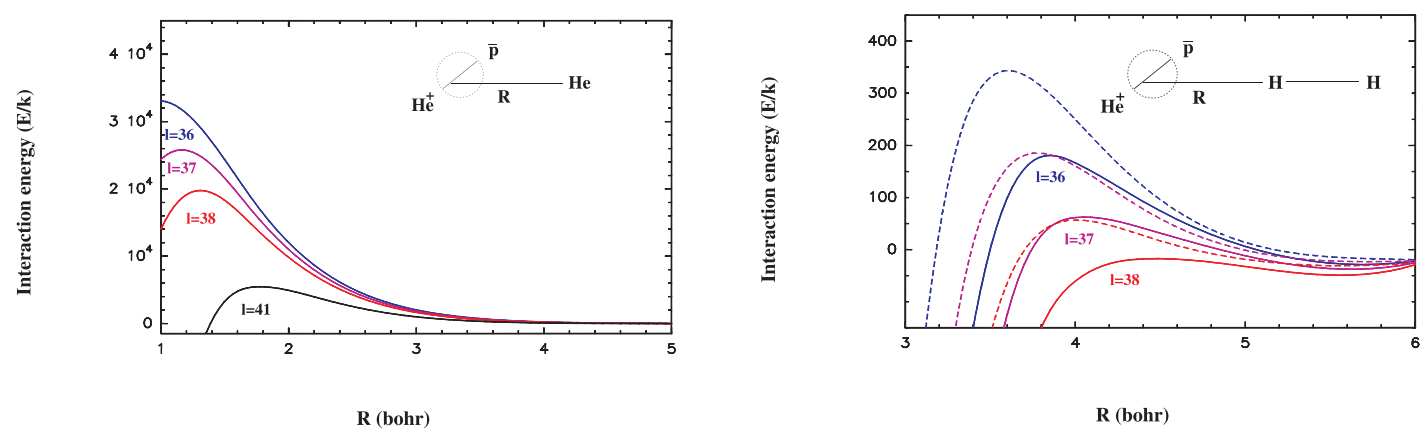

Fig. 2. Interaction energy of $\overline{\mathrm{p}} \mathrm{He}^{+}-\mathrm{He}$ (left) and $\overline{\mathrm{p}} \mathrm{He}^{+}-\mathrm{H}_{2}$ (right) for a $\overline{\mathrm{p}}$ captured on different circular orbits of angular momentum $l$. Energies are expressed on a thermodynamic scale after division by the Boltzman constant $k$. The collisional stability in pure $\mathrm{He}$ is attributed to the existence of high activation barriers. For $\mathrm{H}_{2}$, comparing barriers resulting from an averaging of the electronic potential either over the ro-vibronic quantum states of the nuclei ( $p, \bar{p})$ (continuous lines) or over their classical orbits (dotted lines) shows that quantum corrections strongly lower barriers at low temperatures.

In contrast, the rapid destruction by $\mathrm{H}_{2}$ is due to very low activation barriers, for which the quantum ro-vibronic delocalization of light $(\mathrm{p}, \overline{\mathrm{p}})$ nuclei cannot be ignored [5]. Those barriers can also be crossed at low temperature by tunneling. One sees there that atomcules constitute an interesting benchmark system, which could provide new insights into the physico-chemistry of cold terrestrial and interstellar molecules and radicals, such as $\mathrm{OH}$ or $\mathrm{NH}_{3}$. Atomcules are thus of interest also for chemists and astrophysicists.

During my work with Pierre, we also studied other physico-chemistry aspects such as the possible synthesis of anti-hydrogen $\left(\overline{\mathrm{H}}=\overline{\mathrm{p}} \mathrm{e}^{+}\right)$by dissociative recombination of atomcules with positronium $\left(\mathrm{Ps}=\mathrm{e}^{+} \mathrm{e}^{-}\right)$[6], the existence of other metastable atomcules and the confinement of antiprotons in nanometric cages (fullerenes). For more details, please see my doctoral dissertation [7].

\section{References}

1. Yamazaki et al. Nature, 361, 238. (1993)

2. Widmann et al. Phys. Rev. A, 53, 3129 (1996)

3. Korobov Phys. Rev. A, 54, 1749. (1996)

4. Sauge et al. Chem. Phys., 265, 47 (2001)

5. Sauge et al. Chem. Phys., 283, 433 (2002)

6. Sauge et al. Chem. Phys. Lett., 334, 330 (2001)

7. Sauge "Physico-Chimie des Atomcules d'Hélium antiprotonique: Modélisation de Processus réactifs en présence d'Antimatière", doctoral thesis, Université Joseph Fourier (2000) 